\title{
The Approximate Solution of High-Order Nonlinear Ordinary Differential Equations by Improved Collocation Method with Terms of Shifted Chebyshev Polynomials
}

\author{
Yalçın Öztürk ${ }^{1}$ - Mustafa Gülsu²
}

Published online: 11 July 2015

C) Springer India Pvt. Ltd. 2015

\begin{abstract}
In this paper, we present a direct computational method for solving the higher-order nonlinear differential equations by using collocation method. This method transforms the nonlinear differential equation into the system of nonlinear algebraic equations with unknown shifted Chebyshev coefficients, via Chebyshev-Gauss collocation points. The solution of this system yields the Chebyshev coefficients of the solution function. The method is valid for both initial-value and boundary-value problems. Several examples are presented to illustrate the accuracy and effectiveness of the method by the approximate solutions of very important equations of applied mathematics such as Lane-Emden equation, Riccati equation, Van der Pol equation. The approximate solutions can be very easily calculated using computer program Maple 13.
\end{abstract}

Keywords Nonlinear differential equation - Shifted Chebyshev polynomial - Collocation method · Lane-Emden equations · Riccati equations · Van der Pol equation

\section{Introduction}

Non-linear differential equations are indispensable tools for modelling many physical phenomenon such as chemical reactions, spring-mass systems, and beam bending. These equations are also useful in ecology and economics. The most popular of this type equations are Riccati, Emden-Fowler, Duffing, Van der Pol, Rayleigh and Yermakov's equations. Most nonlinear differential equations do not have exact solutions, so approximation and numerical techniques must be used. Recently, several numerical methods to solve nonlinear differen-

Yalçın Öztürk

yozturk@mu.edu.tr

Mustafa Gülsu

mgulsu@mu.edu.tr

1 Ula Ali Koçman Vocational School, Mugla Sttkı Koçman University, Mugla, Turkey

2 Department of Mathematics, Faculty of Science, Mugla Sıtkı Koçman University, Mugla, Turkey 
tial equations have been given such as Adomian decomposition method [1,2], linearization method [3], a sixth-degree B-spline method [4], the decomposition method [5]. Moreover, a Homotopy perturbation method (HPM) [6] has been proposed to solve a quadratic Riccati equation. A quasilinearization approach to the nonlinear problems in physics has been discussed in [7]. Chebshev collocation matrix method [8] has been presented the numerical solution of nonlinear differential equations. The method in [8] transforms the nonlinear differential equation into the matrix equation, which corresponds to a system of nonlinear algebraic equations with unknown Chebyshev coefficients, via Chebyshev collocation points.

In this paper, it is concerned with the application of shifted Chebyshev collocation method to obtain the numerical solution of nonlinear differential equation of the form:

$$
\sum_{k=0}^{m} \sum_{s=0}^{n} Q_{k, s}(x) y^{s}(x) y^{(k)}(x)+\sum_{k=1}^{m} \sum_{s=1}^{m} P_{k, s}(x) y^{(s)}(x) y^{(k)}(x)=f(x)
$$

with conditions

$$
\sum_{k=0}^{m-1}\left(a_{i k} y^{(k)}(a)+b_{i k} y^{(k)}(b)+c_{i k} y^{(k)}(c)\right)=\alpha_{i}, \quad i=0,1, \ldots, m-1 .
$$

where $y^{(0)}(x)=y(x)$ and $y(x) \in C^{m}[0, L]$ is an unknown function. $Q_{k, s}(x), P_{k, s}(x)$ and $f(x)$ known functions which are defined on $[0, L]$.

We seek the approximate solution of Eq. (1) with Eq. (2) as the truncated shifted Chebyshev series defined by

$$
y_{N}(x)=\sum_{r=0}^{N} a_{r} T_{L, r}^{*}(x), \quad x \in[0, L]
$$

where $N$ is any positive integer and $N>m$ and $T_{L, r}^{*}(x), r=0,1, \ldots, N$ denote the shifted Chebyshev polynomials which can be obtained with the aid of the following recurrence formula $[9,10]$ :

$$
T_{L, r+1}^{*}(x)=2\left(\frac{2 x}{L}-1\right) T_{L, r}^{*}(x)-T_{L, r-1}^{*}(x), r=1,2, \ldots
$$

where $T_{L, 0}^{*}(x)=1, T_{L, 1}^{*}(x)=\frac{2 x}{L}-1$. The analytic form of the shifted Chebyshev polynomilas $T_{L, r}^{*}(x)$ of degree $r$ is given by

$$
T_{L, r}^{*}(x)=r \sum_{p=0}^{r}(-1)^{r-p} \frac{(r+p-1) ! 2^{2 p}}{(r-p) !(2 p) ! L p} x^{p}
$$

where $T_{L, r}^{*}(0)=(-1)^{r}$ and $T_{L, r}^{*}(L)=1$. The orthogonality condition is

$$
\int_{0}^{L} T_{L, j}^{*}(x) T_{L, i}^{*}(x) w_{L}(x) d x=h_{k} \delta_{j i}
$$

where $w_{L}(x)=\left(L x-x^{2}\right)^{-1 / 2}$ and $h_{i}=b_{i} \pi / 2, b_{0}=2, b_{i}=1, k \geq 1$. By Eq. (4), we have the $k$-th derivatives of $T_{L, r}^{*}(x)$

$$
\left(T_{L, r}^{*}\right)^{(k)}(x)=T_{L, r}^{*, k}(x)=r \sum_{p=m}^{r}(-1)^{r-p} p(p-1) \ldots(p-k+1) \frac{(r+p-1) ! 2^{2 p}}{(r-p) !(2 p) ! L^{k}} x^{p-k}
$$

where $p \geq m-1$. 


\section{Solution Method}

Using Eq. (3), we have the $k$-th derivatives of approximate solution $y_{N}(x)$

$$
y_{N}^{(k)}(x)=\sum_{r=0}^{N} a_{r}\left(T_{L, r}^{*}\right)^{(k)}(x)=\sum_{r=k}^{N} a_{r} T_{L, r}^{*, k}(x)
$$

From (1), (3) and (6), we have

$$
\begin{aligned}
& \sum_{k=0}^{m} \sum_{s=0}^{n} Q_{k, s}(x)\left(\sum_{r=0}^{N} a_{r} T_{L, r}^{*}(x)\right)^{s}\left(\sum_{r=k}^{N} a_{r} T_{L, r}^{*, k}\right) \\
& +\sum_{k=1}^{m} \sum_{s=1}^{m} P_{k, s}(x)\left(\sum_{r=s}^{N} a_{r} T_{L, r}^{*, s}\right)\left(\sum_{r=k}^{N} a_{r} T_{L, r}^{*, k}\right)=f(x)
\end{aligned}
$$

We now collocate Eq. (7) at $N-m+1$ points $x_{p}, p=0,1, \ldots, N-m$, we obain following equations

$$
\begin{aligned}
& \sum_{k=0}^{m} \sum_{s=0}^{n} Q_{k, s}\left(x_{q}\right)\left(\sum_{r=0}^{N} a_{r} T_{L, r}^{*}\left(x_{q}\right)\right)^{s}\left(\sum_{r=k}^{N} a_{r} T_{L, r}^{*, k}\left(x_{q}\right)\right) \\
& +\sum_{k=1}^{m} \sum_{s=1}^{m} P_{k, s}\left(x_{q}\right)\left(\sum_{r=s}^{N} a_{r} T_{L, r}^{*, s}\left(x_{q}\right)\right)\left(\sum_{r=k}^{N} a_{r} T_{L, r}^{*, k}\left(x_{q}\right)\right)=f\left(x_{q}\right)
\end{aligned}
$$

where $x_{p}$ are roots of $T_{L, m}^{*}(x)$. Also, by substituting Eq. (6) in the conditions Eq. (2) we obtain $k$-times equations as follows:

$$
\begin{aligned}
& \sum_{k=0}^{m-1}\left(a_{i k} \sum_{r=k}^{N} a_{r} T_{L, r}^{*, k}(a)+b_{i k} \sum_{r=0}^{N} a_{r} T_{L, r}^{*, k}(b)+c_{i k} \sum_{r=0}^{N} a_{r} T_{L, r}^{*, k}(c)\right) \\
& \quad=\alpha_{i}, i=0,1, \ldots, m-1 .
\end{aligned}
$$

Equation 8, together with $k$-times equations of conditions Eq. (9) give $(N+1)$ non-linear algebraic equations Solving these nonlinear algebraic systems, we obtain the unknown shifted Chebyshev coefficients $a_{r}, r=0,1, \ldots, N$. Consequently $y_{N}(x)$ to approximate $y(x)$ given in Eq. (1) can be calculated.

\section{Error Analysis}

In this section, we present convergence analysis of the mention method. We assume that $y(x)$ is a sufficiently smooth function on $[0,1]$ and $I_{N}(x)$ is the interpolating polynomial to $y$ at $x_{i}$, where $x_{i}, i=0,1, \ldots, n$ are the Cbebyshev-Gauss grid points, then we have $[9,10]$

$$
y(x)-I_{N}(x)=\frac{y^{(N+1)}(\lambda)}{(N+1) !} \prod_{i=0}^{N}\left(x-x_{i}\right), \lambda \in[0,1]
$$

Therefore, we have [29-32]

$$
\left|y(x)-I_{N}(x)\right| \leq \frac{1}{2^{2 N+1}}\left\|y^{(N+1)}(x)\right\|_{\infty} .
$$


Definition [9] The least-square norm is defined by

$$
\|y\|_{2}=\left(\int_{a}^{b} w(x)|y(x)|^{2} d x\right)^{1 / 2}
$$

where $w(x)$ is a non-negative weight function.

Theorem Suppose that the known functions in Eq. (1) are real $(N+1)$-times continuosly differential functions on the $[0,1]$ and

$$
y_{N}(x)=\sum_{r=0}^{N} a_{r} T_{L, r}^{*}(x)
$$

are the shifted Chebyshev polynomials expansion of the exact solution.

Let

$$
\overline{y_{N}}(x)=\sum_{r=0}^{N} \overline{a_{r}} T_{L, r}^{*}(x)
$$

be the approximate solution obtained by proposed method, then there exist real number $\alpha$ such that

$$
\left\|y(x)-y_{N}(x)\right\|_{2} \leq \alpha \frac{1}{2^{N+1}}\left\|y^{(N+1)}(x)\right\|_{\infty}+\sqrt{\frac{3 \pi}{8}}\|A-\bar{A}\|_{2}
$$

where

$$
A=\left[a_{0} a_{1} \cdots a_{N}\right] \text { and } \bar{A}=\left[\overline{a_{0}} \overline{a_{1}} \ldots \overline{a_{N}}\right] .
$$

Proof Let $y_{N}(x)$ is real-valued polynomials of degree $\leq N$ and $y_{N}(x)$ is the best aprroximation of $y(x)$. We can write

$$
\left\|y(x)-y_{N}(x)\right\|_{2} \leq\left\|y(x)-\overline{y_{N}}(x)\right\|_{2}+\left\|\overline{y_{N}}(x)-y_{N}(x)\right\|_{2}
$$

Using Eq. (11), we obtain

$$
\begin{aligned}
\left\|y(x)-y_{N}(x)\right\|_{2}= & \left(\int_{0}^{1}\left|y(x)-y_{N}(x)\right|^{2} d x\right)^{1 / 2} \\
& \leq\left(\int_{0}^{1}\left[\frac{1}{2^{N+1}(N+1) !}\left\|y^{(N+1)}(x)\right\|_{\infty}\right]^{2} d x\right)^{1 / 2} \\
= & \sqrt{L} \frac{1}{2^{2 N+1}(N+1) !}\left(\left\|y^{(N+1)}(x)\right\|_{\infty}\right)^{N+1}
\end{aligned}
$$

and we have

$$
\left\|y(x)-\overline{y_{N}(x)}\right\|_{2}=\left(\int_{0}^{1}\left[\sum_{r=0}^{N}\left(a_{r}-\overline{a_{r}}\right) T_{r}^{*}(x)\right]^{2} d x\right)^{1 / 2}
$$




$$
\begin{aligned}
& \leq\left(\int_{0}^{1}\left[\sum_{r=0}^{N}\left(a_{r}-\overline{a_{r}}\right)^{2}\right]\left[\sum_{r=0}^{N}\left|T_{r}^{*}(x)\right|^{2}\right] d x\right)^{1 / 2} \\
= & {\left[\sum_{r=0}^{N}\left(a_{r}-\overline{a_{r}}\right)^{2}\right]^{1 / 2}\left(\sum_{r=0}^{N} \int_{0}^{1}\left|T_{r}^{*}(x)\right|^{2} d x\right)^{1 / 2}=\sqrt{\frac{3 \pi}{8}}\|A-\bar{A}\| . }
\end{aligned}
$$

Moreover, we can easly check the accuracy of the method. Since the truncated Chebyshev series (3) is an approximate solutions of Eq. (1), when the function $y_{j}^{N}(x), j=0,1, \ldots, m$ and its first derivatives are substituted in Eq. (1) the resulting equation must be satisfied approximately[32]; that is, for $x_{i} \in[0,1], i=0,1,2, \ldots$

$$
\left|\sum_{k=0}^{m} \sum_{s=0}^{n} Q_{k, s}\left(x_{i}\right) y^{s}\left(x_{i}\right) y^{(k)}\left(x_{i}\right)+\sum_{k=1}^{m} \sum_{s=1}^{m} P_{k, s}\left(x_{i}\right) y^{(s)}\left(x_{i}\right) y^{(k)}\left(x_{i}\right)-f\left(x_{i}\right)\right| \cong 0
$$

On the other hand, the error can be estimated by the function [10]

$$
E_{N}(x)=\sum_{k=0}^{m} \sum_{s=0}^{n} Q_{k, s}(x) y^{s}(x) y^{(k)}(x)+\sum_{k=1}^{m} \sum_{s=1}^{m} P_{k, s}(x) y^{(s)}(x) y^{(k)}(x)-f(x) .
$$

\section{Numerical Results}

We show the efficienciy of the present method using the following examples. In Tables, $N_{e}=\left|y(x)-y_{N}(x)\right|$ is absolute error for selected points.

Example 1 Consider the following Riccati differential equation $[6,11]$ :

$$
y^{\prime}(x)-2 y(x)+y^{2}(x)=1
$$

with initial conditions

$$
y(0)=0 .
$$

Exact solution of this problem is

$$
y(x)=1+\sqrt{2} \tanh \left(\sqrt{2} x+\frac{1}{2} \log \left(\frac{\sqrt{2}-1}{\sqrt{2}+1}\right)\right) .
$$

Let us suppose that $y(x)$ is approximated by a truncated Chebyshev series in the form

$$
y_{6}(x)=\sum_{i=0}^{6} a_{i} T_{1, r}^{*}(x), x \in[0,1]
$$

Using Eq. (8), we have

$$
\sum_{r=1}^{6} a_{r} T_{1, r}^{*, 1}\left(x_{q}\right)-2 \sum_{r=0}^{6} a_{r} T_{1, r}^{*}\left(x_{q}\right)+\left(\sum_{r=0}^{6} a_{r} T_{1, r}^{*}\left(x_{q}\right)\right)^{2}=1
$$


with $q=0,1,2, \ldots, 5$, where $x_{q}$ are roots of the shifted Chebyshev polynomials $T_{1,6}^{*}(x)$

$$
\begin{aligned}
& x_{0}=\frac{1}{2}-\frac{1}{2} \cos \left(\frac{\pi}{14}\right), \quad x_{1}=\frac{1}{2}-\frac{1}{2} \cos \left(\frac{3 \pi}{14}\right), \quad x_{2}=\frac{1}{2}-\frac{1}{2} \cos \left(\frac{5 \pi}{14}\right), \\
& x_{3}=\frac{1}{2}, \quad x_{4}=\frac{1}{2}+\frac{1}{2} \cos \left(\frac{5 \pi}{14}\right), \quad x_{5}=\frac{1}{2}+\frac{1}{2} \cos \left(\frac{3 \pi}{14}\right)
\end{aligned}
$$

and

$$
y(0)=a_{0}-a_{1}+a_{2}-a_{3}+a_{4}-a_{5}+a_{6}=0
$$

Thus, we obtain seven nonlinear algebraic equations with the seven unknown by Eqs. 14-15. Now solving equations, we obtain the coefficients:

$$
\begin{aligned}
& a_{0}=0.803725, \quad a_{1}=0.873802, \quad a_{2}=0.044314, \quad a_{3}=-0.019645, \\
& a_{4}=-0.0037358, \quad a_{5}=0.001331, \quad a_{6}=0.000291 .
\end{aligned}
$$

This coefficients are substituting into Eq. (13), we get the approximate solution for $N=6$

$$
\begin{aligned}
y_{6}(x)= & 0.999923 x+1.003816 x^{2}+0.287515 x^{3}-0.0879134 x^{4} \\
& -1.110271 x^{5}+0.597349 x^{6}
\end{aligned}
$$

This problem was solved by HPM [6] and Adomian decomposition method (ADM) [11]. In Table 1, we give the comparision of the absolute errors HPM with 9 terms, ADM with 6

\begin{tabular}{|c|c|c|c|c|c|c|c|}
\hline \multirow[t]{2}{*}{$\mathrm{x}$} & \multirow[t]{2}{*}{ Exact solution } & \multicolumn{2}{|l|}{ PM } & \multicolumn{2}{|l|}{ ADM } & \multicolumn{2}{|l|}{ HPM } \\
\hline & & $\mathrm{N}=6$ & $\mathrm{~N}_{\mathrm{e}}=6$ & $\begin{array}{l}\text { Numerical } \\
\text { solution }\end{array}$ & $\begin{array}{l}\text { Absolute } \\
\text { errors }\end{array}$ & $\begin{array}{l}\text { Numerical } \\
\text { solution }\end{array}$ & $\begin{array}{l}\text { Absolute } \\
\text { errors }\end{array}$ \\
\hline 0.0 & 0.000000 & 0.000000 & $0.000 \mathrm{E}-0$ & 0.000000 & $0.000 \mathrm{E}-0$ & 0.000000 & $0.000 \mathrm{E}-0$ \\
\hline 0.1 & 0.110295 & 0.110298 & $0.350 \mathrm{E}-5$ & 0.1102678 & $0.271 \mathrm{E}-4$ & 0.110295 & $0.340 \mathrm{E}-7$ \\
\hline 0.2 & 0.241976 & 0.241979 & $0.291 \mathrm{E}-5$ & 0.241642 & $0.334 \mathrm{E}-3$ & 0.241972 & $0.445 \mathrm{E}-5$ \\
\hline 0.3 & 0.395104 & 0.395108 & $0.395 \mathrm{E}-5$ & 0.393924 & $0.118 \mathrm{E}-2$ & 0.395027 & $0.775 \mathrm{E}-4$ \\
\hline 0.4 & 0.567812 & 0.567807 & $0.421 \mathrm{E}-5$ & 0.565631 & $0.218 \mathrm{E}-2$ & 0.567223 & $0.588 \mathrm{E}-3$ \\
\hline 0.5 & 0.756014 & 0.755998 & $0.161 \mathrm{E}-4$ & 0.754166 & $0.184 \mathrm{E}-2$ & 0.753190 & $0.282 \mathrm{E}-2$ \\
\hline 0.6 & 0.953566 & 0.953572 & $0.676 \mathrm{E}-5$ & 0.955968 & $0.240 \mathrm{E}-2$ & 0.943490 & $0.100 \mathrm{E}-0$ \\
\hline 0.7 & 1.159489 & 1.153000 & $0.515 \mathrm{E}-5$ & 1.166675 & $0.137 \mathrm{E}-1$ & 1.123821 & $0.291 \mathrm{E}-1$ \\
\hline 0.8 & 1.346363 & 1.346357 & $0.590 \mathrm{E}-5$ & 1.381290 & $0.349 \mathrm{E}-1$ & 1.274579 & $0.717 \mathrm{E}-1$ \\
\hline 0.9 & 1.526911 & 1.526793 & $0.118 \mathrm{E}-3$ & 1.594332 & $0.674 \mathrm{E}-1$ & 1.371096 & $0.155 \mathrm{E}-0$ \\
\hline 1.0 & 1.689498 & 1.690420 & $0.921 \mathrm{E}-\mathrm{E}-3$ & 1.799999 & $0.110 \mathrm{E}-0$ & 1.384920 & $0.304 \mathrm{E}-0$ \\
\hline
\end{tabular}
terms and Present method for $N=6$. Moreover, the numerical results and the absolute errors are compared in Figs. 1 and 2 respectively. Numerical results show that the Present method is more accurate than the others methods.

Example 2 Consider a fifth order nonlinear differential equation [8]:

$$
y^{(5)}(x)-e^{-x} y^{2}(x)=0
$$

Table 1 Numerical result for Example 1 


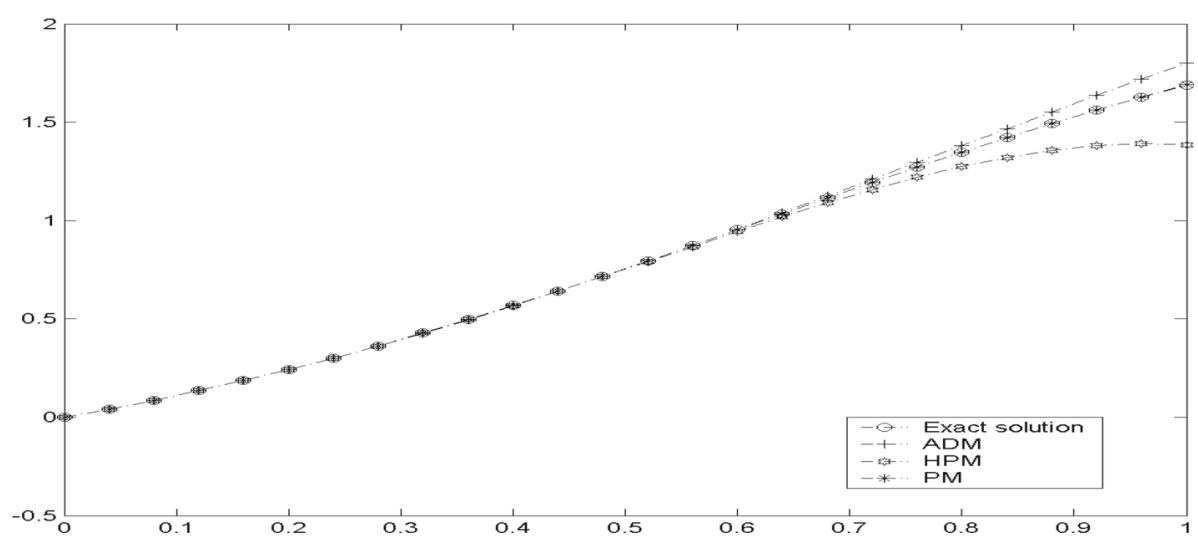

Fig. 1 Comparision of numerical solutions for ADM, HPM and present method

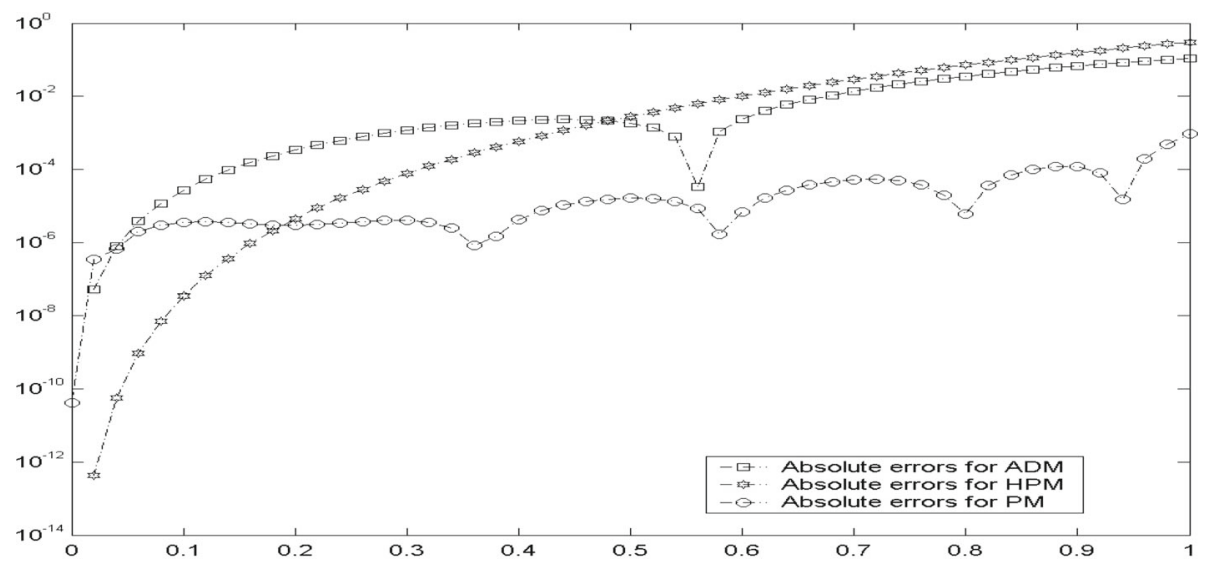

Fig. 2 Comparision of absolute errors for numerical methods ADM, HPM and PM

subject to the boundary conditions $y(0)=y^{\prime}(0)=y^{\prime \prime}(0)=1, y(1)=y^{\prime}(1)=e$ and the exact solution is $y(x)=e^{x}$. Taking $N=6,10$, we obtain the approximate solutions

$$
\begin{aligned}
y_{6}(x)= & 1+x+0.5 x^{2}+0.166726 x^{3}+0.041533 x^{4}+0.008163 x^{5}+0.001558 x^{6} \\
y_{10}(x)= & 1+x+0.5 x^{2}+0.166666 x^{3}+0.041666 x^{4}+0.083333 x^{5}+0.00138892 x^{6} \\
& +0.198269 E-3 x^{7}+0.250859 E-4 x^{8}+0.246432 E-5 x^{9}+0.421894 E-6 x^{10}
\end{aligned}
$$

Comparision of the absolute errors obtained by Chebyshev collocation matrix method (CCMM) and Present method are given in Table 2.

Example 3 Let us consider following the Lane-Emden equation

$$
y^{\prime \prime}(x)+\frac{\alpha}{x} y^{\prime}(x)+g(x, y)=h(x)
$$

with initial conditions

$$
y(0)=\alpha_{0}, y^{\prime}(0)=\alpha_{1}
$$


Table 2 Numerical result for Example 2

\begin{tabular}{|c|c|c|c|c|}
\hline \multirow[t]{2}{*}{$\mathrm{x}$} & \multicolumn{2}{|l|}{ PM } & \multicolumn{2}{|l|}{ CCMM } \\
\hline & $\mathrm{N}_{\mathrm{e}}=6$ & $\mathrm{~N}_{\mathrm{e}}=10$ & $\mathrm{~N}_{\mathrm{e}}=6$ & $\mathrm{~N}_{\mathrm{e}}=10$ \\
\hline 0.0 & $0.10 \mathrm{E}-13$ & $0.00 \mathrm{E}-00$ & $0.00 \mathrm{E}-0$ & $0.20 \mathrm{E}-15$ \\
\hline 0.1 & $0.45 \mathrm{E}-7$ & $0.30 \mathrm{E}-13$ & $0.80 \mathrm{E}-7$ & $0.20 \mathrm{E}-12$ \\
\hline 0.2 & $0.23 \mathrm{E}-6$ & $0.90 \mathrm{E}-13$ & $0.40 \mathrm{E}-6$ & $0.10 \mathrm{E}-11$ \\
\hline 0.3 & $0.41 \mathrm{E}-6$ & $0.35 \mathrm{E}-12$ & $0.10 \mathrm{E}-5$ & $0.20 \mathrm{E}-11$ \\
\hline 0.4 & $0.24 \mathrm{E}-6$ & $0.12 \mathrm{E}-11$ & $0.20 \mathrm{E}-5$ & $0.50 \mathrm{E}-11$ \\
\hline 0.5 & $0.49 \mathrm{E}-6$ & $0.10 \mathrm{E}-11$ & $0.30 \mathrm{E}-5$ & $0.70 \mathrm{E}-11$ \\
\hline 0.6 & $0.16 \mathrm{E}-5$ & $0.67 \mathrm{E}-12$ & $0.40 \mathrm{E}-5$ & $0.90 \mathrm{E}-11$ \\
\hline 0.7 & $0.27 \mathrm{E}-5$ & $0.18 \mathrm{E}-11$ & $0.40 \mathrm{E}-5$ & $0.10 \mathrm{E}-10$ \\
\hline 0.8 & $0.27 \mathrm{E}-5$ & $0.83 \mathrm{E}-12$ & $030 \mathrm{E}-5$ & $0.10 \mathrm{E}-10$ \\
\hline 0.9 & $0.13 \mathrm{E}-5$ & $0.26 \mathrm{E}-12$ & $0.10 \mathrm{E}-5$ & $0.60 \mathrm{E}-11$ \\
\hline 1.0 & $0.10 \mathrm{E}-13$ & $0.00 \mathrm{E}-00$ & $0.20 \mathrm{E}-1$ & $0.30 \mathrm{E}-15$ \\
\hline
\end{tabular}

A discussion of the formulation of these models and the physical structure of the solutions can be found in the literature. For example, for $g(x, y)=y^{n}(x), h(x)=0$, Eq. (16) is the standart Lane-Emden equation that was used to model the thermal behavior of a spherical cloud of gas acting under the mutual attraction of its molecules and subject to the classical laws of thermodynamics and for $g(x, y)=e^{y}, h(x)=0$, Eq. (16) is the isothermal gas sphere equation, where the temperature remains constant [12-20].

Case I Let us consider $\alpha=2, g(x, y)=y(x), h(x)=6+12 x+x^{2}+x^{3}$ and $y(0)=$ $0, y^{\prime}(0)=0$ in Eq. (16). We applied the given method in this paper, we have

$$
\sum_{r=1}^{3} a_{r} T_{1, r}^{*, 2}\left(x_{q}\right)-\frac{2}{x} \sum_{r=0}^{3} a_{r} T_{1, r}^{*, 1}\left(x_{q}\right)+\sum_{r=0}^{3} a_{r} T_{1, r}^{*}\left(x_{q}\right)=6+12 x_{p}+x_{p}^{2}+x_{p}^{3}
$$

and substituting roots of $T_{1,2}^{*}(x)$ into Eq. (18), we obtain

$$
\begin{aligned}
& a_{0}+58.846787 a_{1}-190.351251 a_{2}+275.138438 a_{3}=6.808635 \\
& a_{0}+8 a_{1}+15 a_{2}-24 a_{3}=12.375
\end{aligned}
$$

with conditions

$$
\begin{aligned}
& a_{0}-a_{1}+a_{2}-a_{3}=0 \\
& 2 a_{1}-8 a_{2}+18 a_{3}=0 .
\end{aligned}
$$

Therefore, it is obtained that 4 linear algebraic equations with 4 unknown. Solving above linear equations, we obtain the coefficients

$$
a_{0}=0.6875 \quad a_{1}=0.96875 \quad a_{2}=0.3125 \quad a_{3}=0.03125
$$

Then so approximate solution is obtained for $N=3$

$$
y_{3}(x)=x^{3}+x^{2}
$$

which is the exact solution.

Case II Taking $g(x, y)=y^{3}(x), h(x)=0$ and $y(0)=1, y^{\prime}(0)=0$. The solution to this initial value problem is well known to be $y(x)=\left(1+\frac{x^{2}}{3}\right)^{-1 / 2}$. In Table 3 , we give the absolute errors for various $N$ and these results are plotted in Fig. 3. 
Table 3 Error values of Case II in Example 3

\begin{tabular}{lllll}
\hline $\mathrm{x}$ & $\mathrm{N}_{\mathrm{e}}=6$ & $\mathrm{~N}_{\mathrm{e}}=7$ & $\mathrm{~N}_{\mathrm{e}}=8$ & $\mathrm{~N}_{\mathrm{e}}=9$ \\
\hline 0.2 & $0.433 \mathrm{E}-6$ & $0.172 \mathrm{E}-8$ & $0.551 \mathrm{E}-8$ & $0.904 \mathrm{E}-9$ \\
0.4 & $0.146 \mathrm{E}-5$ & $0.183 \mathrm{E}-7$ & $0.844 \mathrm{E}-8$ & $0.189 \mathrm{E}-8$ \\
0.6 & $0.444 \mathrm{E}-6$ & $0.956 \mathrm{E}-7$ & $0.970 \mathrm{E}-8$ & $0.264 \mathrm{E}-8$ \\
0.8 & $0.119 \mathrm{E}-5$ & $0.173 \mathrm{E}-6$ & $0.167 \mathrm{E}-7$ & $0.800 \mathrm{E}-9$ \\
1.0 & $0.623 \mathrm{E}-5$ & $0.499 \mathrm{E}-6$ & $0.588 \mathrm{E}-7$ & $0.117 \mathrm{E}-7$ \\
\hline
\end{tabular}

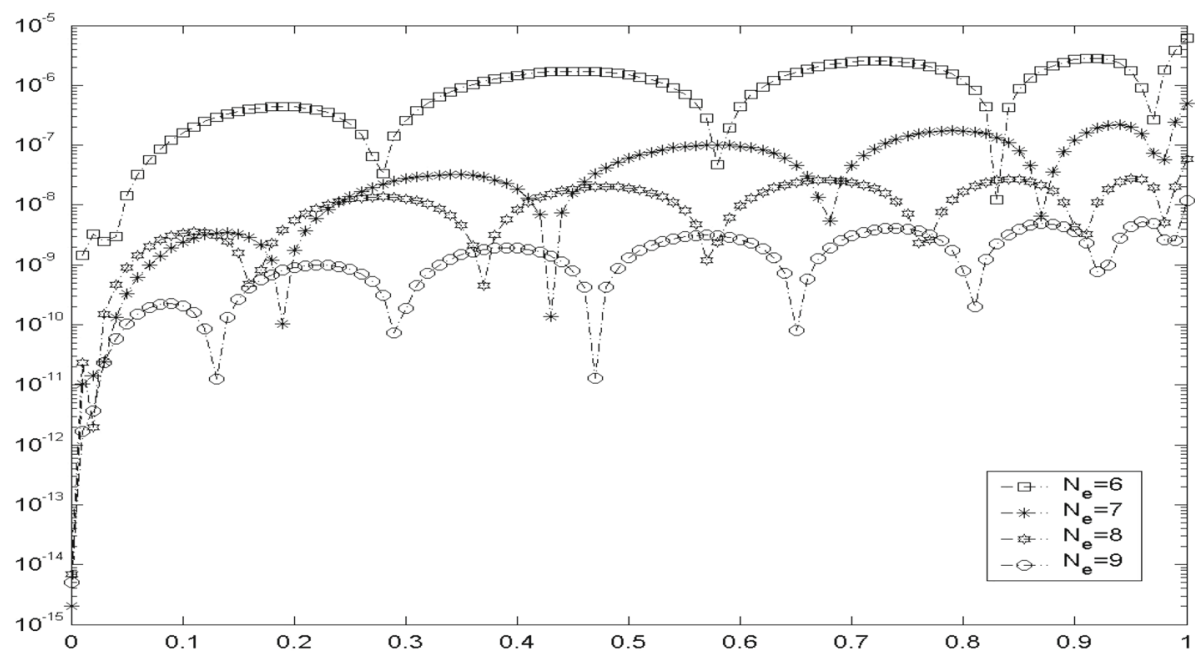

Fig. 3 Comparision of absolute errors of Case II in Example 3

Table 4 Numerical results for CaseIII in Example 3

\begin{tabular}{lllllll}
\hline $\mathrm{x}$ & Exact solution & \multicolumn{2}{l}{$\mathrm{PM}(\mathrm{N}=9)$} & & $\mathrm{HCM}$ & \\
& & Approximate values & Absolute errors & Approximate values & Absolute errors \\
\hline 0.00 & 0.0000000000 & 0.000000000 & $0.000 \mathrm{E}-0$ & & 0.0000000000 & $0.000 \mathrm{E}-0$ \\
0.01 & -0.0001999900 & -0.0001999905 & $0.598 \mathrm{E}-9$ & -0.0001970587 & $0.293 \mathrm{E}-5$ \\
0.10 & -0.0199006617 & -0.0199005895 & $0.721 \mathrm{E}-7$ & -0.0198967225 & $0.394 \mathrm{E}-5$ \\
0.50 & -0.4462871026 & -0.4462872851 & $0.182 \mathrm{E}-6$ & -0.4462840851 & $0.302 \mathrm{E}-5$ \\
1.00 & -1.3862943611 & -1.3862955089 & $0.114 \mathrm{E}-5$ & -1.3862934297 & $0.931 \mathrm{E}-6$ \\
\hline
\end{tabular}

Case III In this case, taking $g(x, y)=4\left(2 e^{y}+e^{y / 2}\right), h(x)=0, \alpha=2$ and $y(0)=$ $0, y^{\prime}(0)=0$. In this case Eq. (16) has the exact solution $y(x)=-2 \ln \left(1+x^{2}\right)$. This type of equation has been solved by [16] with Hermite collocation method (HCM). We apply our method to solve Eq. (16) with Case III. Then, Table 4 shows the comparison of exact solution and HCM obtained by the new method proposed in this paper. In order to compare the present method with exact solution, the resulting graph of Case III is shown in Fig. 4 for various $N$.

Example 4 Let us consider the Van der Pol equation [8,21,22]

$$
y^{\prime \prime}(x)-0.05\left(1-y^{2}(x)\right) y^{\prime}(x)+y(x)=0
$$




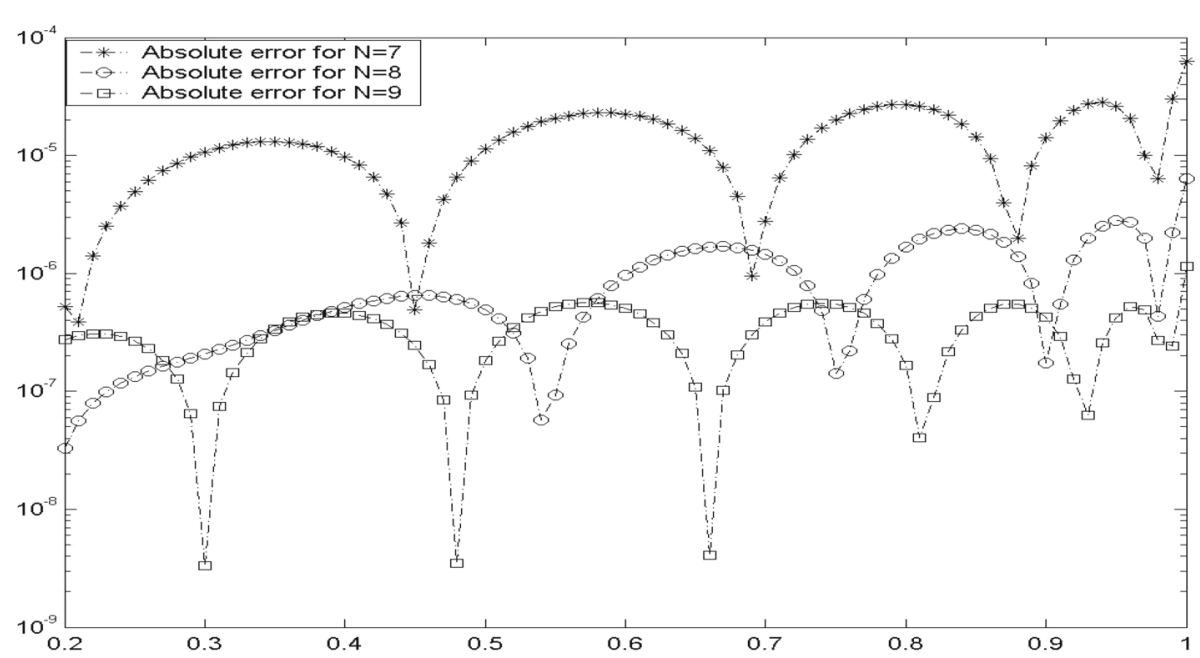

Fig. 4 Comparision of absolute errors of Case III in Example 3

Table 5 Comparison of absolute errors for some numerical methods for Example 4

\begin{tabular}{lllll}
\hline$x$ & CCMM & Runge-Kutta method & Gear method & Present method \\
\hline 0.0 & 0 & 0 & 0 & 0 \\
0.1 & 0.0500416561 & 0.0500416616 & 0.0500416561 & 0.0500416560 \\
0.2 & 0.0998321663 & 0.0998321792 & 0.0998321668 & 0.0998321667 \\
0.3 & 0.1488697052 & 0.1488697072 & 0.1488697063 & 0.1488697063 \\
0.4 & 0.1966564148 & 0.1966564313 & 0.1966564167 & 0.1966564165 \\
0.5 & 0.2427036829 & 0.2427036961 & 0.2427036728 & 0.2427036725 \\
0.6 & 0.2865373683 & 0.2865373842 & 0.2865373709 & 0.2865373708 \\
0.7 & 0.3277031549 & 0.3277032041 & 0.3277031577 & 0.3277031578 \\
0.8 & 0.3657715085 & 0.3657715349 & 0.3657715117 & 0.3657715113 \\
0.9 & 0.4003425800 & 0.4003426519 & 0.4003425836 & 0.4003425833 \\
1.0 & 0.4310507066 & 0.4310507546 & 0.4310507104 & 0.4310507094 \\
\hline
\end{tabular}

with conditions $y(0)=0, y^{\prime}(0)=0.5$. For comparision the solutions at the selected points, Eq. (19) is solved by CCMM [8], Runga Kutta method [21] and Gear single-step extrapolation method [22]. Numerical results are given in Table 5 and numerical results are plotted in Fig. 5. From Fig. 5 and Table 5, We can see that the numerical results are the same line.

Example 5 Consider the nonlinear diferential equation

$$
\begin{aligned}
& y^{\prime \prime \prime}(x)+y^{\prime \prime}(x)+y^{\prime}(x) y(x)=-e^{-2 x} \\
& y(0)=1, \quad y^{\prime}(0)=-1, y^{\prime \prime}(0)=1
\end{aligned}
$$

with exact solution $y(x)=e^{-x}$. In Table 6 , we compare the maximum absolute errors and $L_{2}$ errors for various $N$. These results are the similar values. 


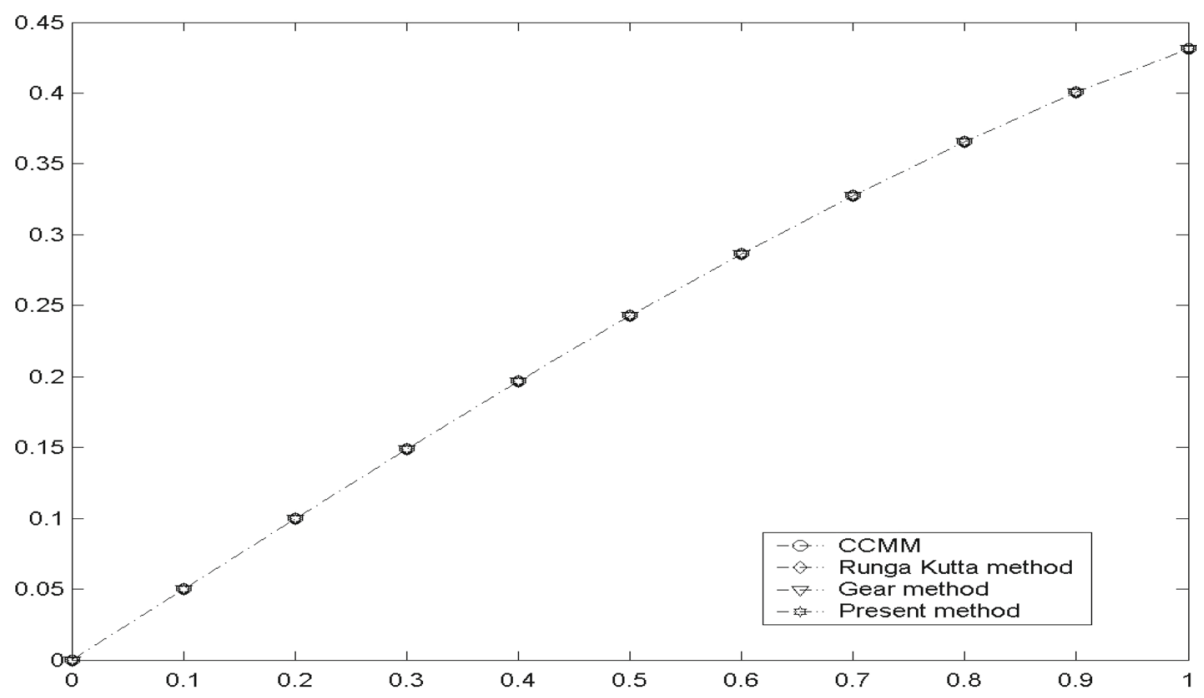

Fig. 5 Comparision of numerical methods for Example 4

Table 6 Numerical result for Example 5

\begin{tabular}{lll}
\hline Present method & Maximum absolute errors & $L_{2}$ errors \\
\hline$N=5$ & $0.119252 \times 10^{-4}$ & $0.704723 \times 10^{-5}$ \\
$N=8$ & $0.445095 \times 10^{-6}$ & $0.158113 \times 10^{-6}$ \\
$N=10$ & $0.524856 \times 10^{-12}$ & $0.424679 \times 10^{-12}$ \\
\hline
\end{tabular}

\begin{tabular}{lll}
\hline Present method & Maximum absolute errors & $L_{2}$ errors \\
\hline$N=6$ & $0.516389 \times 10^{-4}$ & $0.238789 \times 10^{-4}$ \\
$N=8$ & $0.695539 \times 10^{-7}$ & $0.269757 \times 10^{-7}$ \\
$N=10$ & $0.665694 \times 10^{-10}$ & $0.866025 \times 10^{-9}$ \\
$N=12$ & $0.425280 \times 10^{-13}$ & $0.417133 \times 10^{-12}$ \\
\hline
\end{tabular}

Table 7 Numerical result for Example 6

Example 6 Consider the following nonlinear diferential equation with initial conditions:

$$
\begin{aligned}
& y^{(4)}(x)+y^{\prime \prime \prime}-y^{\prime \prime}+\left(1-y^{2}(x)\right) y^{\prime}=x^{3} \cos ^{2}(x) \sin (x) \\
& -x^{2} \cos ^{3}(x)+2 x \cos (x)-2 \cos (x)+6 \sin (x) \\
& y(0)=0, \quad y^{\prime}(0)=1, \quad y^{\prime \prime}(0)=0, \quad y^{\prime \prime \prime}(0)=-3
\end{aligned}
$$

The exact solution of this problem $y=x \cos (x)$. To obtained numerical results maximum absolute errors and $L_{2}$ errors are compare in Table 7. In Fig. 6, we display the absolute errors for various $N$. 


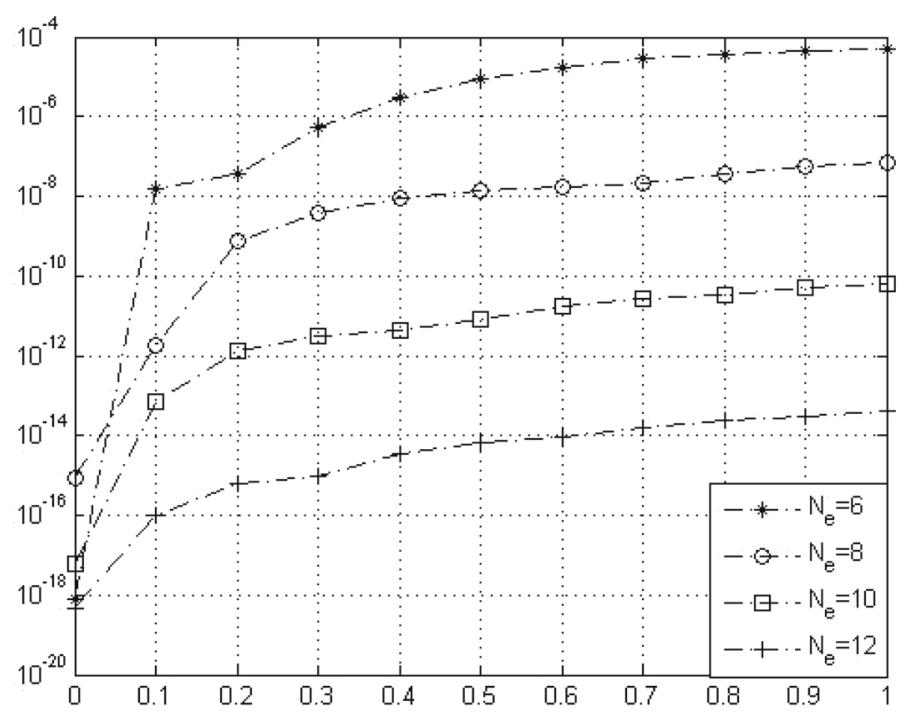

Fig. 6 Comparision of absolute error for Example 6

\section{Conclusion}

In this article, we present the effective numerical method for solving nonlinear differential equations. The present method provides a reliable technique which gives highly accurate results and requires less work than traditional techniques and existing numerical methods.

In Examples, we get numerical solutiom of the important nonlinear differential equations such as Riccati equations, Emden-Fowler equations, Van der Pol equations. An valuable feature of this method is that the analytical solution is obtained as demonstrated in Example 3 when the exact solution is polynomial. Moreover, Tables and Figures show that this method gives better approximate solutions than the other methods.

\section{References}

1. Wazwaz, A.M.: A new method for solving initial value problems in second-order ordinary differential equations. Appl. Math. Comput. 128, 45-57 (2002)

2. Adomian, G.: Nonlinear Stochastic Operator Equations. Academic Press, San Diego (1986)

3. Ramos, J.I.: Linearization techniques for singular initial-value problems of ordinary differential equations. Appl. Math. Comput. 161, 525-542 (2005)

4. Caglar, H.N., Caglar, S.H., Twizell, E.H.: The numerical solution of fifth-order boundary value problems with sixth-degree B-Spline functions. Appl. Math. Lett. 12, 25-30 (1999)

5. Wazwaz, A.M.: The numerical solution of fifth-order boundary value problems by the decomposition method. J. Comput. Appl. Math. 136(1-2), 259-270 (2001)

6. Abbasbandy, S.: Homotopy perturbation method for quadratic Riccati differential equation and comparison with Adomian's decomposition method. Appl. Math. Comput. 172, 485-490 (2006)

7. Mandelzweig, W.B., Tabakin, F.: Quasilinearization approach to nonlinear problems in physics with application to nonlinear ODEs. Comput. Phys. Commun. 141, 268-281 (2001)

8. Daşçığlu, A., Yaslan, H.: The solution of high-order nonlinear ordinary differential equations by Chebshev series. Appl. Math. Comput. 217, 5658-5666 (2011)

9. Mason, J.C., Handscomb, D.C.: Chebyshev Polynomials. Chapman and Hall/CRC, New York (2003)

10. Body, J.P.: Chebyshev and Fourier Spectral Methods. University of Michigan, New York (2000) 
11. Adomian, G.: Solving Frontier Problems of Physics: Decomposition Method. Kluwer Academic Publishers, Dordrecth (1994)

12. Agarwala, R.P., O’Reganb, D.: Second order initial value problems of Lane-Emden type. Appl. Math. Lett. 20, 1198-1205 (2007)

13. Biles, D.C., Robinson, M.P., Spraker, J.S.: A generalization of the Lane-Emden equation. J. Math. Anal. Appl. 273, 654-666 (2002)

14. Shawagfeh, N.T.: Nonperturbative approximate solution for Lane-Emden equation. J. Math. Phys. 34, 4364-4369 (1993)

15. Davis, H.T.: Introduction to Nonlinear Differential and Integral Equations. Dover, New York (1962)

16. Parand, K., Dehghan, M., Rezaei, A.R., Ghaderi, S.: An approximation algorithm for the solution of the nonlinear Lane-Emden type equations arising in astrophysics using Hermite functions collocation method. Comput. Phys. Commun. 181, 1096-1108 (2010)

17. Singh, O.P., Pandey, R.K., Singh, V.K.: An analytic algorithm of Lane-Emden type equations arising in astrophysics using modified Homotopy analysis method. Comput. Phys. Comm. 180, 1116-1124 (2009)

18. Yıldırım, A., Öziş, T.: Solutions of singular IVPs of Lane-Emden type by the variational iteration method. Nonlinear Anal. 70, 2480-2484 (2009)

19. Hasan, Y.Q., Zhu, L.M.: Solving singular boundary value problems of higher-order ordinary differential equations by modified Adomian decomposition method. Commun. Nonlinear Sci. Numer. Simul. 14, 2592-2596 (2009)

20. Kumar, M., Singh, N.: Modified Adomian decomposition method and computer implementation for solving singular boundary value problems arising in various physical problems. Comput. Chem. Eng. 34(11), 1750-1760 (2010)

21. Vigo-Aguiar, J., Ramos, H.: Variable stepsize implementation of multistep methods for $y$ ” $(x)=f(x, y$, y'). J. Comput. Appl. Math. 192(1), 114-131 (2006)

22. Sheng Cai, Dong, Oyagi, Akira, Abe, Kanji: Parametric excitation of computational mode of the leapfrog scheme applied to the Van der Pol equation. J. Comput. Phys. 107, 146-151 (1993) 